\title{
Improved soft fusion-based cooperative spectrum sensing using particle swarm optimization
}

\author{
Mohsen Akbari ${ }^{1 \mathrm{a})}$, Mohsen Riahi Manesh ${ }^{1}$, Ayman A. El-Saleh ${ }^{1,2}$, \\ and Mahamod Ismail ${ }^{2}$ \\ ${ }^{1}$ Faculty of Engineering, Multimedia University, Jalan Multimedia, \\ 63100 Cyberjaya, Selangor, Malaysia \\ ${ }^{2}$ Department of Electrical, Electronics, and System Engineering, \\ Universiti Kebangsaan Malaysia, 43600 Bangi, Selangor, Malaysia \\ a)mohsen.akbari61@yahoo.com
}

\begin{abstract}
In soft-decision fusion- (SDF-) based cooperative spectrum sensing, weighting the coefficients vector is the main factor affecting the detection performance of cognitive radio networks. In this paper, the use of particle swarm optimization (PSO) algorithm as a prominent technique is proposed to optimize the weighting coefficients vector. The proposed PSO-based scheme opts for the best weighting coefficients vector, leading to improved detection performance of the system. The performance of the proposed method is analyzed and compared with genetic algorithm- (GA-) based technique as well as other conventional SDF schemes through computer simulations. Simulation results validate the robustness of the proposed method over all other SDF techniques.
\end{abstract}

Keywords: spectrum sensing, cognitive radio, PSO, SDF

Classification: Wireless circuits and devices

\section{References}

[1] S. Haykin, "Cognitive radio: Brain-empowered wireless communications," IEEE J. Sel. Areas Commun., vol. 23, no. 2, pp. 201-220, Feb. 2005.

[2] W. Zhang, R. K. Mallik, and K. B. Letaief, "Cooperative spectrum sensing optimization in cognitive radio networks," Proc. IEEE Int. Conf. Commun., pp. 3411-3415, 2008.

[3] J. Ma, G. Zhao, and Y. Li, "Soft combination and detection for cooperative spectrum sensing in cognitive radio networks," IEEE Trans. Wireless Commun., vol. 7, pp. 4502-4507, 2008.

[4] Z. Quan, S. Cui, and A. H. Sayed, "Optimal linear cooperation for spectrum sensing in cognitive radio networks," IEEE J. Sel. Topics Signal Processing, vol. 2, pp. 28-40, 2008.

[5] B. Shen and K. S. Kwak, "Soft combination schemes for cooperative spectrum sensing in cognitive radio networks," ETRI Journal, vol. 31, pp. 263$270,2009$. 
[6] A. A. El-Saleh, M. Ismail, and M. A. M. Ali, "Genetic algorithm-assisted soft fusion-based linear cooperative spectrum sensing," IEICE Electron. Express, vol. 8, no. 18, pp. 1527-1533, 2011.

[7] J. Kennedy and R. Eberhart, "Particle swarm optimization," Proc. IEEE Int. Conf., vol. 4, pp. 1942-1948, 1995.

\section{Introduction}

The electromagnetic radio spectrum is a rare natural resource, the efficient use of which is of extreme importance. To prevent significant interference between different systems, usually, the spectrum bands are statically allocated to specific services. Consequently, only portions of the spectrum are heavily utilized. Cognitive radios (CRs) determine the frequency bands unutilized by licensed system, or primary user (PU), and intelligently exploit it based on spectrum observations [1]. Cooperative spectrum sensing can be classified into two main categories of hard-decision fusion (HDF) [2] and soft-decision fusion (SDF) [3, 4, 5]. In [3], equal gain combination (EGC) and maximal ratio combination (MRC) schemes have been investigated as a solution for weighting the coefficients vector of all CRs in a linear SDF-based system. However, these methods are not optimal since allocated weights to different users are all equal in the EGC method or are merely based on users' received signal-to-noise ratio (SNR) in the MRC technique. In $[4,5]$, the idea of maximizing the normal deflection coefficient (NDC) and modified deflection coefficient (MDC) to optimize the weighting coefficients vector, was proposed. Nevertheless, the mentioned methods lead to sub-optimal results, imposing some performance deteriorations. In [6], the SDF-based cooperative sensing using genetic algorithm (GA) was proposed. Although the GA-based method provides a good solution for weighting the coefficients, it suffers from slow convergence and sub-optimality due to its reliance on binary encoding of a naturally-continuous domain. In this paper, the use of particle swarm optimization (PSO) algorithm is proposed for SDF-based cooperative spectrum sensing to optimize the detection performance of the system. Unlike GA, PSO algorithm deals with continuous variables and does not require binarydecimal encoding/decoding. In addition, there is lesser number of adjusting parameters for the PSO algorithm than those of the GA.

\section{System model}

Fig. 1 demonstrates the block diagram of SDF-based cooperative spectrum sensing in which a fusion center (FC) receives statistical measurements from $M$ secondary users (SUs) in the CR network. SUs operate as relays because they simply amplify and forward the PU signal to FC. The final decision on the presence of $\mathrm{PU}$ is made by the FC as it conducts SDF-based linear weighted calculations on the received SUs signals.

At each SU, the sensing job can be expressed as the binary hypothesis 


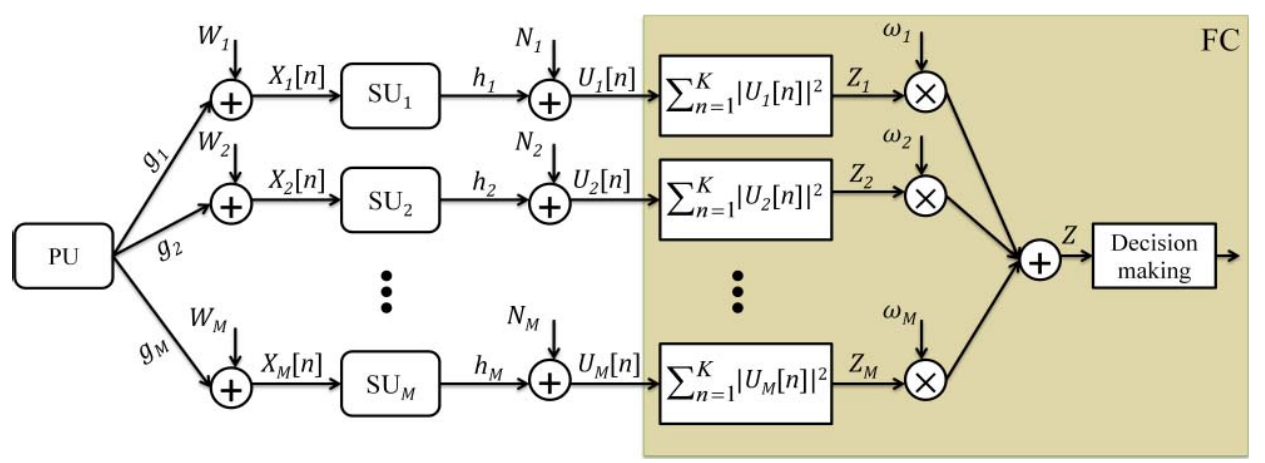

Fig. 1. Block diagram of the cooperative spectrum sensing

test,

$$
\begin{aligned}
& \text { Absence } \rightarrow H_{0}: X_{i}[n]=W_{i}[n] \\
& \text { Presence } \rightarrow H_{1}: X_{i}[n]=g_{i} S[n]+W_{i}[n]
\end{aligned}
$$

where $X_{i}[n]$ is the received sampled signal at $i^{\text {th }}$ SU receiver and $i=1,2, \ldots$, $M, n=1,2, \ldots, K, K=2 B T_{s}$ is the total number of samples of the received signal in which $B$ is the signal bandwidth and $T_{s}$ is the sensing time, $g_{i}$ is the gain of the channel between $\mathrm{PU}$ and $i^{\text {th }} \mathrm{SU}, S[n]$ is the transmitted signal from PU which is considered as an independent and identically distributed (i.i.d.) Gaussian random process with zero mean and variance $\sigma_{S}^{2}$ i.e. $S[n] \sim$ $\mathcal{N}\left(0, \sigma_{S}^{2}\right)$, and $W_{i}[n]$ is additive white Gaussian noise (AWGN) of $i^{\text {th }}$ sensing channel (PU-SU) with zero mean and variance $\sigma_{W_{i}}^{2}$ i.e. $W_{i}[n] \sim \mathcal{N}\left(0, \sigma_{W_{i}}^{2}\right)$. The final test statistic $Z$ calculated by FC before decision making block can be represented by $Z=\sum_{i=1}^{M} \omega_{i} Z_{i}$ where $Z_{i}=\sum_{n=1}^{K}\left|U_{i}[n]\right|^{2}$ is the energy collected by FC from the $i^{\text {th }} \mathrm{SU}$ signal and $U_{i}[n]=\sqrt{P_{R, i}} h_{i} X_{i}[n]+N_{i}[n]$ is the corresponding received signal at FC in which $P_{R, i}$ is the transmit power of $i^{\text {th }} \mathrm{SU}$ and $h_{i}$ is the channel gain between $i^{\text {th }} \mathrm{SU}$ and FC. It is assumed that the reporting channel (SU-FC) noise $N_{i}[n]$ is AWGN with zero mean and variance $\delta_{i}^{2}$ i.e. $N_{i}[n] \sim \mathcal{N}\left(0, \delta_{i}^{2}\right)$. Finally, $\omega_{i}$ is the weighting coefficient of the $i^{\text {th }}$ path. Since all random variables $\left\{Z_{i}\right\}$ are normally distributed, the resultant test statistic $Z$ has also normal distribution with parameters as follows [4]:

$$
\begin{gathered}
E\left(Z \mid H_{0}\right)=\sum_{i=1}^{M} \omega_{i} K \sigma_{0, i}^{2} \\
E\left(Z \mid H_{1}\right)=\sum_{i=1}^{M} \omega_{i} K \sigma_{1, i}^{2} \\
\operatorname{var}\left(Z \mid H_{0}\right)=\sum_{i=1}^{M} 2 \omega_{i}^{2} K\left(\sigma_{0, i}^{2}+\delta_{i}^{2}\right)^{2}=\overrightarrow{\boldsymbol{\omega}}^{T} \Phi_{\mathrm{H}_{0}} \overrightarrow{\boldsymbol{\omega}} \\
\operatorname{var}\left(Z \mid H_{1}\right)=\sum_{i=1}^{M} 2 \omega_{i}^{2} K\left(\sigma_{1, i}^{2}+\sigma_{0, i}^{2}\right)^{2}=\overrightarrow{\boldsymbol{\omega}}^{T} \Phi_{\mathrm{H}_{1}} \overrightarrow{\boldsymbol{\omega}}
\end{gathered}
$$

where $\sigma_{0, i}^{2}=P_{R, i}\left|h_{i}\right|^{2} \sigma_{W_{i}}^{2}+\delta_{i}^{2}$ and $\sigma_{1, i}^{2}=P_{R, i}\left|g_{i}\right|^{2}\left|h_{i}\right|^{2} \sigma_{s}^{2}+\sigma_{0, i}^{2}$ are variances of $U_{i}[n]$ under hypotheses $H_{0}$ and $H_{1}$, respectively. $\overrightarrow{\boldsymbol{\omega}}=\left[\omega_{1}, \omega_{2}, \ldots, \omega_{M}\right]^{T}$ denotes the weighting coefficients vector which is to be optimized and the superscript $T$ represents the transpose of the vector. The covariance matrices un$\operatorname{der} H_{0}$ and $H_{1}$ are $\Phi_{H_{0}}=\operatorname{diag}\left(2 K \sigma_{0, i}^{4}\right)$ and $\Phi_{H_{1}}=\operatorname{diag}\left(2 K\left(P_{R, i}\left|g_{i}\right|^{2}\left|h_{i}\right|^{2} \sigma_{s}^{2}+\right.\right.$ 
$\left.\left.\sigma_{0, i}^{2}\right)^{2}\right)$, respectively where $\operatorname{diag}($.$) is square diagonal matrix whose diagonal$ elements are the elements of a given vector. Assuming the energy threshold at FC is $\beta$ then, $Z \underset{H_{0}}{H_{1}} \beta$ demonstrates the likelihood ratio. Therefore, the final probability of detection $P_{d}$ and probability of false alarm $P_{f}$ can be expressed as

$$
\begin{aligned}
& P_{f}=P\left(Z>\beta \mid H_{0}\right)=Q\left(\frac{\beta-E\left(Z \mid H_{0}\right)}{\sqrt{\operatorname{var}\left(Z \mid H_{0}\right)}}\right) \\
& P_{d}=P\left(Z>\beta \mid H_{1}\right)=Q\left(\frac{\beta-E\left(Z \mid H_{1}\right)}{\sqrt{\operatorname{var}\left(Z \mid H_{1}\right)}}\right)
\end{aligned}
$$

Substituting (2) to (5) into (6) and (7) and computing $P_{d}$ in terms of $P_{f}$, we can conclude:

$$
P_{d}(\overrightarrow{\boldsymbol{\omega}})=Q\left(\frac{Q^{-1}\left(\overline{P_{f}}\right) \sqrt{\overrightarrow{\boldsymbol{\omega}}^{T} \Phi_{\mathrm{H}_{0}} \overrightarrow{\boldsymbol{\omega}}}-\overrightarrow{\boldsymbol{\omega}}^{T} \overrightarrow{\boldsymbol{\theta}}}{\sqrt{\overrightarrow{\boldsymbol{\omega}}^{T} \Phi_{\mathrm{H}_{1}} \overrightarrow{\boldsymbol{\omega}}}}\right)
$$

where $Q(x)=\int_{x}^{+\infty} \frac{1}{\sqrt{2 \pi}} e^{-t^{2} / 2} d t, \overrightarrow{\boldsymbol{\theta}}=\left[\theta_{1}, \theta_{2}, \ldots, \theta_{M}\right]^{T}, \theta_{i}=K P_{R, i}\left|g_{i}\right|^{2}\left|h_{i}\right|^{2} \sigma_{s}^{2}$. Equation (8) provides a reliable measure of detection performance in SDFbased cooperative sensing for a fixed set of false alarm probabilities. It is observable that the detection performance is highly dependent on $\overrightarrow{\boldsymbol{\omega}}$. Therefore, the optimal solution is the weighting vector which maximizes $P_{d}$ in (8). However, since any real multiple of the optimal weighting vector can also be considered as the optimal solution, the $\vec{\omega}$ used in this work satisfies the conditions $0<\omega_{i}<1$ and $\sqrt{\sum_{i=1}^{M} \omega_{i}^{2}}=1$ to reduce the search space of the PSO.

\section{Particle swarm optimization-based weighting method}

PSO algorithm [7] is abstracted from social behavior of swarm of fishes and birds. The behavior of these social organizations is emulated by PSO algorithm. Each particle in PSO algorithm functions based on its own knowledge as well as the group knowledge and has two main features: position and velocity. In each iteration, the information about the best position is cooperatively exchanged among the particles. After adequate number of iterations, the algorithm converges to the optimal solution of the objective function.

In this work, the problem is to maximize the objective function $P_{d}(\overrightarrow{\boldsymbol{\omega}})$ in (8). The steps involved in the PSO algorithm are as follows:

Step 1: Considering the number of particles are $N$, initialize the algorithm by randomly generating $N$ numbers of $\overrightarrow{\boldsymbol{\omega}}_{s}=\left[\omega_{1}, \omega_{2}, \ldots, \omega_{M}\right]^{T}$ : $(s=1, \ldots, N)$ in the range of 0 and 1 . For simplicity, the position and velocity of particle $s$ at iteration $j$ are represented by $\overrightarrow{\boldsymbol{\omega}}_{s}^{(j)}$ and $\overrightarrow{\boldsymbol{v}}_{s}^{(j)}$, respectively. Particle velocities are initially set to zero.

Step 2: Evaluate the values of the objective function corresponding to initial particle positions as $P_{d}\left(\overrightarrow{\boldsymbol{\omega}}_{1}^{(0)}\right), P_{d}\left(\overrightarrow{\boldsymbol{\omega}}_{2}^{(0)}\right), \ldots, P_{d}\left(\overrightarrow{\boldsymbol{\omega}}_{N}^{(0)}\right)$.

Step 3: Find the maximum value of the objective function in the step 2 and set its equivalent particle position as the $\mathbf{P}_{\text {best }, 0}$. Set the iteration number $j=1$. 
Step 4: At the $j^{\text {th }}$ iteration, find the velocity of the $s^{\text {th }}$ particle as follows:

$$
\overrightarrow{\boldsymbol{v}}_{s}^{(j)}=\overrightarrow{\boldsymbol{v}}_{s}^{(j-1)}+c_{1} r_{1}\left[\boldsymbol{P}_{\text {best }, j}-\overrightarrow{\boldsymbol{\omega}}_{s}^{(j-1)}\right]+c_{2} r_{2}\left[\boldsymbol{G}_{\text {best }}-\overrightarrow{\boldsymbol{\omega}}_{s}^{(j-1)}\right]
$$

where $c_{1}$ and $c_{2}$ are the learning acceleration coefficients, $r_{1}$ and $r_{2} \sim$ $U(0,1)$ are uniformly distributed random numbers which present stochastic components to the algorithm. $\boldsymbol{P}_{\text {best }, j}$ is the best value of experienced position of the particles at the $j^{\text {th }}$ iteration. Global best position $\left(\boldsymbol{G}_{\text {best }}\right)$ is the best value of experienced position among all iterations.

Step 5: Update the $s^{\text {th }}$ particle position at the $j^{\text {th }}$ iteration using:

$$
\overrightarrow{\boldsymbol{\omega}}_{s}^{(j)}=\overrightarrow{\boldsymbol{\omega}}_{s}^{(j-1)}+\overrightarrow{\boldsymbol{v}}_{s}^{(j)}
$$

Evaluate the values of objective function corresponding to new particle positions as $P_{d}\left(\overrightarrow{\boldsymbol{\omega}}_{1}^{(j)}\right), P_{d}\left(\overrightarrow{\boldsymbol{\omega}}_{2}^{(j)}\right), \ldots, P_{d}\left(\overrightarrow{\boldsymbol{\omega}}_{N}^{(j)}\right)$.

Step 6: Find the maximum value of the objective function in the step 5 and set its equivalent particle position as the $\boldsymbol{P}_{b e s t, j}$. If $\boldsymbol{P}_{b e s t, j} \geq \boldsymbol{G}_{b e s t}$, replace $\boldsymbol{G}_{\text {best }}$ with $\boldsymbol{P}_{\text {best }, j}$.

Step 7: If the algorithm is converged to a stable value, stop the process. Otherwise, set the iteration number as $j=j+1$ and repeat from step 4 .

\section{Simulation results}

In this work, the proposed PSO-based SDF technique has been implemented in MATLAB and evaluated and compared with GA-based and conventional NDC-, MDC-, MRC- and EGC-based SDF methods. Following the work in [4], we randomly generate the channel gains $\left\{g_{i}, h_{i}\right\}$ and noises $\left\{\sigma_{W_{i}}^{2}, \delta_{i}^{2}\right\}$ which eventually affect the system performance. Table I demonstrates the overall simulation parameters used in this paper. The channel noises and gains pertaining to every CR user are chosen to realize low SNR condition (SNR $<-10 \mathrm{~dB})$ at FC to confirm the effectiveness of the proposed algorithm. Since the channel is considered to be a slow fading channel, $\left\{g_{i}\right\}$ and $\left\{h_{i}\right\}$ are assumed to be constant over every sensing time interval. The

Table I. Simulation parameters

\begin{tabular}{|l|c|}
\hline \hline \multicolumn{2}{|c|}{ CR parameters } \\
\hline \hline Number of users $M$ & 20 \\
\hline Bandwidth $B$ & $6 \mathrm{MHz}$ \\
\hline Sensing time $T_{S}$ & $25 \mu \mathrm{sec}$ \\
\hline SU transmit power $P_{R, i}$ & $33 \mathrm{dBm}$ \\
\hline PU transmit power $\sigma_{S}{ }^{2}$ & $35 \mathrm{dBm}$ \\
\hline PU-SU channel noise $\sigma_{W_{i}}^{2}$ & $20 \leq \sigma_{W_{i}}^{2} \leq 30 \mathrm{dBm}$ \\
\hline SU-FC channel noise $\delta_{i}{ }^{2}$ & $20 \leq \delta_{i}{ }^{2} \leq 30 \mathrm{dBm}$ \\
\hline channel gains $g_{i}$ & $10 \leq g_{i} \leq 20 \mathrm{dBm}$ \\
\hline channel gains $h_{i}$ & $10 \leq h_{i} \leq 20 \mathrm{dBm}$ \\
\hline The step size of $P_{f}\left(0 \leq P_{f} \leq 1\right)$ & 0.01 \\
\hline \hline & PSO algorithm parameters \\
\hline \hline Number of particles $N$ & 25 \\
\hline learning coefficients $c_{1}, c_{2}$ & $c_{1}=c_{2}=2$ \\
\hline
\end{tabular}




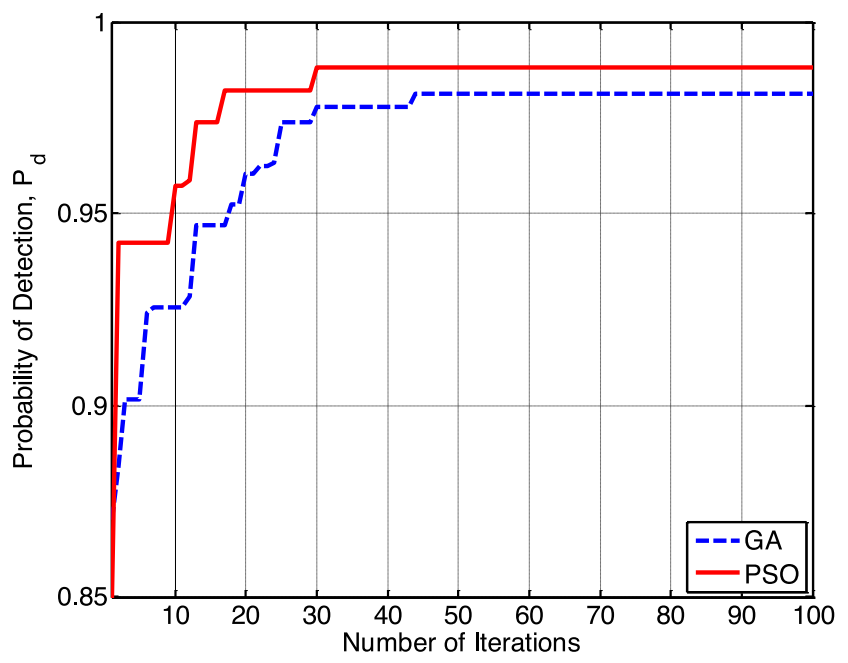

(a)

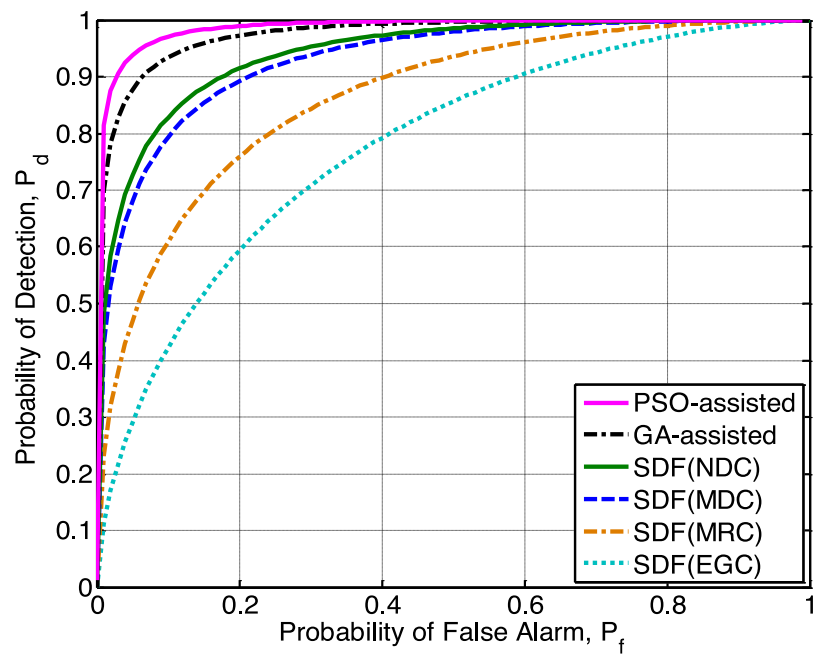

(b)

Fig. 2. (a) Comparison of probability of detection over 100 iterations for PSO and GA, (b) Comparison of probability of detection versus probability of false alarm for PSO-assisted, GA-assisted and other SDF-based schemes

convergence comparison between PSO- and GA-based schemes for a given $P_{f}=0.25$ are shown in Fig. 2 (a). As it can be seen, the PSO-based method converges after 30 iterations while the convergence for GA-based technique is attained after 44 iterations. This implies the fast convergence of the proposed PSO algorithm. An approximate improvement of $32 \%$ in convergence speed of PSO-based method is obvious which confirms the suitability of the algorithm for real time applications.

The optimal weights obtained by PSO and GA algorithms in Fig. 2 (a) are used to plot the receiver operating characteristics (ROC) curve shown in Fig. 2(b) which illustrates the probability of detection of PSO-based, GA-based and all other conventional schemes for different given probabili- 
ties of false alarm. It is observable that PSO-based method outperforms all other methods with a large difference which validates the robustness of our proposed technique. For instance, for the fixed probability of false alarm $P_{f}=0.1$, the probability of detection achieved by PSO is $97 \%$ which is the highest among all.

\section{Conclusion}

A main challenge facing cooperative spectrum sensing schemes in CR networks is the proper selection of the weighting coefficient of each SU and consequently, techniques to optimize these coefficients are vital to the overall detection performance of the system. In this paper, an improved SDF cooperative sensing method based on PSO algorithm has been proposed. The proposed method has been extensively compared with all other conventional techniques such as GA-, NDC- and MRC-based methods. Simulation results indicate that the proposed scheme outperforms all other SDF-based schemes with stationary convergence speed.

\section{Acknowledgments}

The authors are very grateful and express their thanks to the Malaysian Government for funding this work under UKM-OUP-ICT-36-185/2011 researchuniversity operation grant. 